\title{
Estimation of the Maximal Heart Rate to Improve Online Tonic-Clonic Seizure Detection using ECG
}

\author{
Thomas De Cooman ${ }^{1,2}$, Anouk Van de Vel ${ }^{3}$, Berten Ceulemans ${ }^{3,4}$, Lieven Lagae ${ }^{4,5}$, Wim Van \\ Paesschen $^{6}$, Bart Vanrumste ${ }^{1,2,7}$, Sabine Van Huffel ${ }^{1,2}$ \\ ${ }^{1}$ Department of Electrical Engineering (ESAT), STADIUS, KU Leuven, Belgium \\ 2 iMinds Medical IT, Belgium \\ ${ }^{3}$ Department of Neurology-Paediatric Neurology, University Hospital, Antwerp University, Belgium \\ ${ }^{4}$ Rehabilitation Center for Children and Youth Pulderbos, Belgium \\ ${ }^{5}$ Department of Child Neurology, University Hospital, KU Leuven, Belgium \\ ${ }^{6}$ Department of Neurology, University Hospital, KU Leuven, Belgium \\ ${ }^{7}$ Department of Electrical Engineering (ESAT), Advanced Integrated Sensing (AdvISe), KU Leuven, \\ Belgium
}

\begin{abstract}
Previous studies have shown that during several types of seizures, the heart rate increases strongly towards a maximal patient-specific epileptic heart rate $H R_{e p}$. This ictal peak heart rate is one of the most important features for classifying epileptic heart rate increases. We therefore try to estimate $H R_{e p}$, which is done by using least squares support vector machines. The found estimation had a mean square error of 18bpm, which is an improvement compared to age-based estimators. Adding this information to an online seizure detector led to an increased performance (F1-score: $14.65 \%$ to $18.72 \%$ ) with a decreased detection delay (23.8s to 11.9s).
\end{abstract}

\section{Introduction}

Since the last couple of decades, online automated epileptic seizure detection at home has become of increased interest [1]. Previous studies showed that complex partial and tonic-clonic seizures are often accompanied with a strong ictal heart rate increase (HRI, see figure 1) [2]. This makes ECG analysis a possible option for online seizure detection at home.

To improve the usability of these algorithms, patientindependent algorithms are required in practice. This causes a big problem as the heart rate (HR) characteristics from the ictal HRI strongly depend on the specific patient. One of the most useful features for patient-specific seizure detection is the reached peak heart rate $H R_{p}$ [3]. Typically, epileptic HRIs will lead to larger peak heart rates due to a longer sympathetic activation compared to non- epileptic HRIs (see figure2). If the seizure lasts sufficiently long, the HR will reach a certain maximal epileptic HR value $H R_{e p}$ which it does not exceed during the seizure. For seizures within one patient, $H R_{e p}$ typically reaches similar values with possible small interseizure changes .

In this paper, we try to make an estimation $\widetilde{H R}_{e p}$ of $H R_{e p}$ each time a strong HRI is occurring. The estimation $\widetilde{H R}_{e p}$ is obtained by using information prior to the point in time where the strong HRI is detected (at point $t_{\nabla H} R^{*}$, see figure 1). In case of a sufficiently long seizure, the HRI should then lead to a $H R_{p}$ close to $\widetilde{H R}_{e p}$, whereas for non-epileptic HRIs $H R_{p}$ should be significantly lower than $\widetilde{H R}_{e p}$. This estimation is then used to improve an existing online seizure detection algorithm [4] by using this estimation as a lower bound for the reached peak HR $H R_{p}$.

In [5], a threshold on the HR is set by estimating the maximal HR of the patient by only using the patient's age as described in [6]. Analysis however shows that this agebased prediction is insufficient for the prediction of $H R_{e p}$. Other algorithms try to avoid HR thresholding by looking for a strong (percentual) heart rate increase compared to the baseline HR $[7,8]$. These parameters however also strongly vary from patient to patient.

\section{Data and methods}

\subsection{Data selection}

The estimation of the maximal epileptic HR $H R_{e p}$ is evaluated on seizures coming from 2 datasets. Dataset 1 contains 25 tonic-clonic seizures from 7 pediatric patients which were recorded at the Pulderbos revalidation cen- 


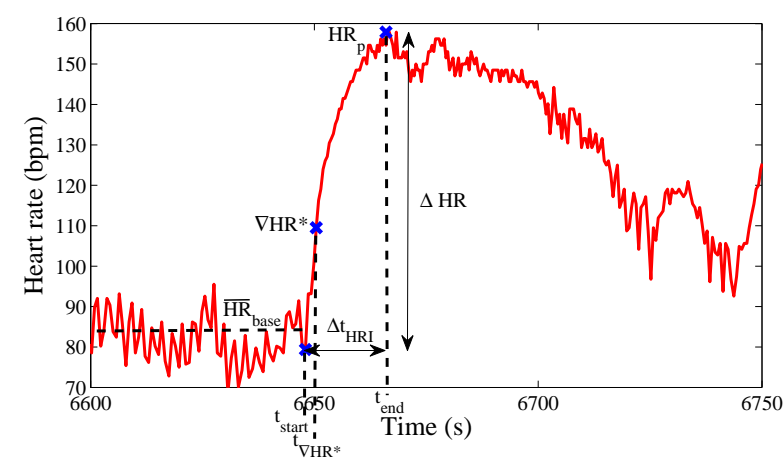

Figure 1. Example of an ictal HRI. The seizure onset is located at $6648 \mathrm{~s}$ in this example.

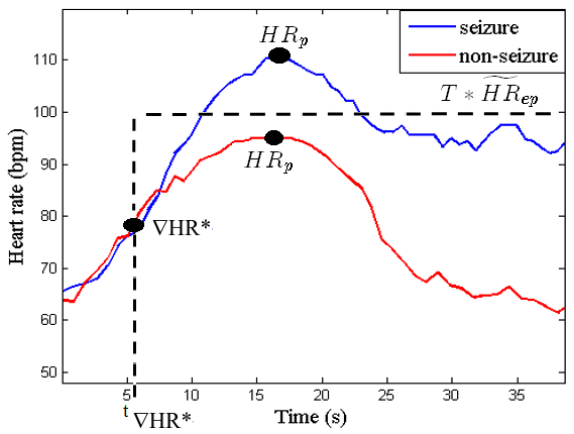

Figure 2. Comparison of a seizure and a non-seizure HRI originating from the same patient.

ter. Dataset 2 contains 127 complex partial seizures (CPS) from 17 temporal lobe epilepsy patients, recorded at the UZ Leuven. Seizures in both datasets were annotated by experts using video-EEG monitoring. The ECG signals were recorded with a sampling frequency of $250 \mathrm{~Hz}$.

Only seizures that last sufficiently long are able to reach their maximal epileptic HR $H R_{e p}$ [3]. Therefore only the noise-free seizures that led to a $H R_{p}$ near the assumed maximal epileptic HR were used for estimating $H R_{e p}$ (so that $H R_{e p}=H R_{p}$ ). These seizures were selected from the datasets after manual inspection of the HR data and expert reports. In total 60 seizures from 20 patients were used for the evaluation of the estimator (see table 1).

Table 1. The used datasets for the estimation of $H R_{e p}$.

\begin{tabular}{r|cccc} 
Set & $\begin{array}{c}\text { Seizure } \\
\text { type }\end{array}$ & Age & \# Patients & $\begin{array}{c}\text { \# Analysed } \\
\text { seizures }\end{array}$ \\
\hline 1 & Tonic-clonic & $3-18$ & 6 & 24 \\
2 & CPS & $9-54$ & 14 & 36
\end{tabular}

The discussed seizure detection algorithms are only evaluated on the entire first dataset, containing in total 252 hours of data recorded during nights.

\subsection{Significant HRI extraction}

In order to detect when a strong HRI is occurring, a previously discussed method is used [4], which works as follows. First the $\mathrm{R}$ peaks are detected by an online $\mathrm{R}$ peak detection algorithm. From the online constructed tachogram, we then compute the HR gradient $\nabla H R$ for each new HR measurement. $\nabla H R$ is computed as the gradient of the linear fit over $10 \mathrm{HR}$ measurements. Whenever a gradient $\nabla H R^{*}$ becomes higher than $1 \mathrm{bpm} / \mathrm{s}$ (at $t_{\nabla H R^{*}}$, see figure 1), we assume a strong HRI is occurring. The start of the HRI can then be retraced by checking when the gradient was negative for the last time (at $t_{\text {start }}$ ), whereas the end of the HRI $\left(t_{e n d}\right)$ is found on-the-run with the detection of the next first negative gradient. A HRI is stated to be significantly strong if the following rules apply (see figure 1 for the definition of the parameters):

$$
\begin{gathered}
\Delta H R>10 \mathrm{bpm} \\
\frac{\Delta H R}{\Delta t_{H R I}}>0.35 \mathrm{bpm} / \mathrm{s} \\
\frac{H R_{p}}{\overline{H R}_{\text {base }}}>1.1
\end{gathered}
$$

The different values of these rules were set heuristically based on a different dataset in [4]. We enhance this algorithm by adding an extra rule

$$
H R_{p}>T * \widetilde{H R}_{e p}
$$

with $\widetilde{H R}_{e p}$ the estimated maximal epileptic HR (at the time of observation) and $T$ a fixed safety percentage to use as a lower bound for the achieved peak heart rate $H R_{p}$.

\subsection{Fast significant HRI extraction}

A faster version of the above mentioned HRI extraction procedure is also evaluated. In this case, once a $\nabla H R^{*}$ is found, every new HR measurement is taken as if it is the end of the HRI. For each new HR measurement after $t_{\nabla H R^{*}}$, rules (1)-(4) are evaluated until all rules are fulfilled or until the end of the HRI is detected. This has as main advantage that the algorithm does not have to wait for the HRI to end, making it able to detect the seizures more quickly than the original version. Due to the addition of rule (4), the algorithm would only alarm if the new HR measurement is sufficiently close to $\widetilde{H R}_{e p}$ (see figure 2 ).

\subsection{Maximal epileptic HR estimation}

The estimation of the maximal epileptic HR is done by solving a regression problem using least-squares support vector machines (LS-SVM) [9], which is formulated as

$$
\min _{w, b, e} \frac{1}{2} w^{T} w+\gamma * \frac{1}{2} \sum_{k=1}^{N} e_{k}^{2}
$$


such that

$$
\begin{aligned}
H R_{e p, k} & =w^{T} \varphi\left(x_{k}\right)+b+e_{k} \\
& =\widetilde{H R}_{e p, k}+e_{k}
\end{aligned}
$$

with $x_{k}$ the extracted features for regression, $H R_{e p, k}$ and $\widetilde{H R}_{e p, k}$ the real and estimated maximal epileptic HR of sample $k$ and $\varphi($.$) the used Gaussian kernel. The estima-$ tor is evaluated on 60 noise-free seizures for which the assumption is made that they achieved their maximal epileptic HR, so that $H R_{e p, k}=H R_{p, k}$ for these seizures.

Extracted features include several pre-ictal HR variability measurements, pre-ictal HR frequency information and $\nabla H R^{*}$. Pre-ictal features are computed on the 60s of HR data before $t_{\text {start }}$. The features of interest are selected automatically using a forward feature selection procedure, adding features to the pool of selected features if they lead to a lower mean square error (MSE). The estimator is evaluated by using leave-one-patient-out cross validation (LOPO CV).

\subsection{Online seizure detection algorithms}

The goal for the estimation of the maximal epileptic HR is to improve online seizure detection in a patientindependent approach. Three seizure detection algorithms are evaluated here (see figure 3 ). As reference algorithm (Method A), the procedure discussed in [4] is used. Features from before and during the significant HRIs are extracted and classified using a SVM classifier. Features were added to the pool of used features if they resulted in an increased performance on the training set. Evaluation of the algorithm is done by using LOPO CV.

Method B enhances method A by including the information of the maximal epileptic HR estimator (rule (4)). Method C differs from method B by using the fast significant HRI extraction procedure discussed in section 2.3.

\section{Results \& discussion}

\subsection{Maximal epileptic HR estimation}

Figure 4 shows both the original and estimated maximal epileptic HR for the inspected seizures. An overall MSE of $\pm 18 \mathrm{bpm}$ is found, with a standard deviation of $13 \mathrm{bpm}$. Most of the estimations have a relative limited error. The estimation $\widetilde{H R}_{e p}$ seems to slightly overestimate most of the low $H R_{e p}$ values. The estimation is also very bad for two seizures with $H R_{e p}$ around 180bpm coming from one adult patient. This might be due to the lack of other seizures with such a high $H R_{e p}$, so that the LS-SVM regressor could not yet fully adept for these values. Introducing more sufficiently long seizures in the training procedure might be able to solve this issue.

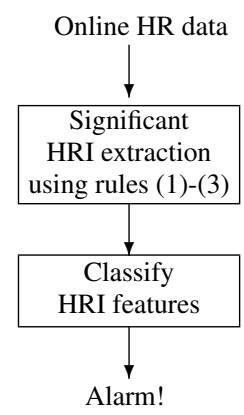

(a)Method A

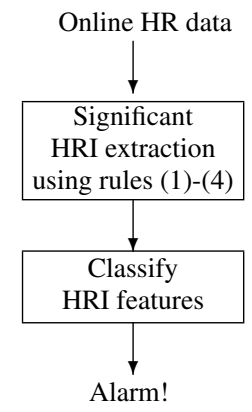

(b)Method B

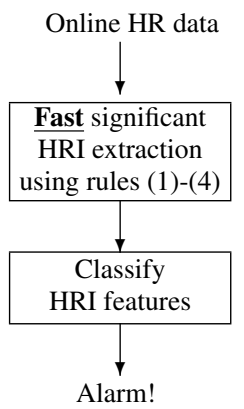

(c)Method C
Figure 3. Overview of the tested online seizure detectors.

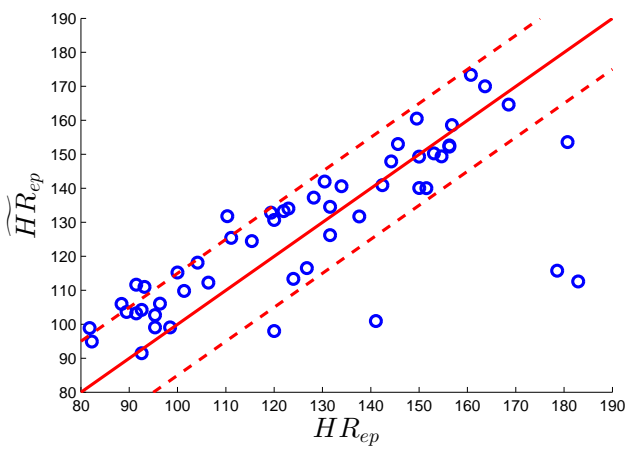

Figure 4. Scatterplot of the real $\left(H R_{e p}\right)$ and estimated $\left(\widetilde{H R}_{e p}\right)$ maximal epileptic heart rates. The dashed lines indicate the average standard error on the estimation.

The best features that were selected for this function estimation were the maximal HR gradient $\nabla H R^{*}$, the preictal mean RR-interval $\overline{R R}_{\text {base }}$ and the feature

$$
\operatorname{Mobility}\left(R R_{\text {base }}^{\prime}\right)-\operatorname{Mobility}\left(R R_{\text {base }}\right)
$$

using Hjorth's mobility parameter on the pre-ictal RRintervals $R R_{\text {base }}$ and the successive differences $R R_{\text {base }}^{\prime}$ [10] . This parameter gives an indication of the complexity of the pre-ictal RR-intervals.

For comparison, an estimation using the age-based maximal HR estimation in [6] leads to a MSE of $\pm 67.5 \mathrm{bpm}$, which is typically a strong overestimation of $H R_{e p}$. This shows that this age-based approach is insufficiently accurate for the intended goal.

\subsection{Online seizure detection}

Table 2 shows the results for the proposed seizure detection algorithms and several algorithms from the literature. The reference method A outperforms other algorithms from the literature on the examined dataset in terms of sensitivity ( $\mathrm{Se}$ ) and false positives/hour (FP/h), but results in a longer detection delay of $23.8 \mathrm{~s}$. 
Table 2. Results for online seizure detection.

\begin{tabular}{r|cccc} 
Method & $\mathrm{Se}(\%)$ & $\mathrm{FP} / \mathrm{h}$ & $\mathrm{F} 1$-score & delay $(\mathrm{s})$ \\
\hline Method A & 92.0 & 1.05 & 14.65 & 23.8 \\
Method B & 88.0 & 0.76 & 18.49 & 24.9 \\
Method C & 88.0 & 0.75 & 18.72 & 11.9 \\
$-\overline{\text { Method [5] }}$ & $-\overline{84} \overline{4} \overline{0}-$ & $-\overline{2} . \overline{6} 2$ & $-\overline{5.95}$ & $-\overline{10 . \overline{3}}--$ \\
Method [7] & 92.0 & 2.01 & 8.27 & 6.8 \\
Method [8] & 96.0 & 2.24 & 7.82 & 6.7
\end{tabular}

Method B is able to further improve the performance of method A by incorporating the information of the $H R_{e p}$ estimation, which can be seen in an improved F1-score

$$
F_{1}=2 * \frac{S e * P P V}{S e+P P V}
$$

with PPV the positive predictive value. $T=0.95$ in rule (4) led to the best performance for both methods B and C.

Method $\mathrm{C}$ leads to a much smaller average detection delay (11.9s) by using the fast significant HRI extraction procedure, while the performance stays nearly the same compared to method B. It seems that after the HR exceeds $T * \widetilde{H R}_{e p}$ during a HRI, the data after this point does not lead to further algorithm improvement for this patient group. Rule (4) allows detection only if the HR is sufficiently close to the estimated maximal epileptic HR $\widetilde{H R}_{e p}$. This causes a few extra seconds of delay compared to the literature, but results in a much lower FP rate, showing that this longer delay is required for a better performance.

It should be noted that the algorithm is only tested here on pediatric patients, which all had $H R_{p}$ above $120 \mathrm{bpm}$. We assume the added value on seizure detection to be even larger when patients with a wider range of age and $H R_{e p}$ are evaluated. This is however left for future work.

\section{Conclusion}

The estimation of the maximal epileptic heart rate using LS-SVM regression showed to correlate very well with the true epileptic peak heart rates. The performance of the discussed seizure detection algorithm improved by incorporating this information. Future work will go into optimizing the estimation further by evaluating more seizures and test the enhanced seizure detection algorithms on data with a more age-varying patient group.

\section{Acknowledgements}

Research supported by Research Council KUL: CoE PFV/10/002 (OPTEC), GOA/10/09 MaNet, PhD/Postdoc grants; FWO: PhD/Postdoc grants, G.0427.10N (Integrated EEG-fMRI), G.0108.11 (Compressed Sensing), G.0869.12N (Tumor imaging), G.0A5513N (Deep brain stimulation); IWT: PhD/Postdoc grants, TBM $110697-$ NeoGuard; iMinds Medical Information Technologies: SBO 2014; Belgian Federal Science Policy Office IUAP P7/19 (DYSCO, Dynamical systems, control and optimization 2012-2017); Belgian Foreign AffairsDevelopment Cooperation: VLIR UOS programs; EU MC ITN TRANSACT 2012 (no 316679), ERC Advanced Grant BIOTENSORS (no 339804), ERASMUS EQR Community service engineer (no 539642-LLP-1-2013); T. De Cooman is supported by an IWT PhD Grant.

\section{References}

[1] Van de Vel A, Cuppens K, Bonroy B, Milosevic M, Jansen K, Van Huffel S, Vanrumste B, Lagae L, Ceulemans B. Non-eeg seizure-detection systems and potential sudep prevention: State of the art. Seizure 2013;22(5):345-355.

[2] Leutmezer F, Schernthaner C, Lurger S, Pötzelberger K, Baumgartner C. Electrocardiographic changes at the onset of epileptic seizures. Epilepsia 2003;44(3):348-354.

[3] De Cooman T, Van de Vel A, Ceulemans B, Lagae L, Vanrumste B, Van Huffel S. Online detection of tonic-clonic seizures in pediatric patients using ecg and low-complexity incremental novelty detection. In 37th Annual International Conference of the IEEE Engineering in Medicine and Biology Society. 2015; in press.

[4] De Cooman T, Carrette E, Boon P, Meurs A, Van Huffel $\mathrm{S}$. Online seizure detection in adults with temporal lobe epilepsy using single-lead ECG. In Proceedings of the 22nd European Signal Processing Conference (EUSIPCO), 2014. IEEE, 2014; 1532-1536.

[5] Ungureanu C, Bui V, Roosmalen W, Aarts RM, Arends JB, Verhoeven R, Lukkien JJ, et al. A wearable monitoring system for nocturnal epileptic seizures. In Medical Information and Communication Technology (ISMICT), 2014 8th International Symposium on. IEEE, 2014; 1-5.

[6] Tanaka H, Monahan KD, Seals DR. Age-predicted maximal heart rate revisited. Journal of the American College of Cardiology 2001;37(1):153-156.

[7] Osorio I. Automated seizure detection using EKG. International Journal of Neural Systems 2014;24(02):1450001.

[8] van Elmpt W, Wouter J, Nijsen T, Griep P, Arends J. A model of heart rate changes to detect seizures in severe epilepsy. Seizure 2006;15(6):366-375.

[9] Suykens JAK, Van Gestel T, De Brabanter J, De Moor B, Vandewalle J. Least Squares Support Vector Machines. World Scientific Pub. Co., 2002.

[10] Hjorth B. EEG analysis based on time domain properties. Electroencephalography and clinical neurophysiology 1970;29(3):306-310.

Address for correspondence:

Thomas De Cooman

Kasteelpark Arenberg 10 box 2446

3001 Leuven, Belgium

thomas.decooman@esat.kuleuven.be 